\title{
The insertion of the anterior horn of the medial meniscus: an anatomic study
}

\section{Ato Ampomah Brown}

Department of Anatomy, School of Medical Sciences, University of Cape Coast, Ghana

Corresponding author:

Ato Ampomah Brown

Department of Anatomy, School of Medical Sciences

University of Cape Coast

Cape Coast, Ghana

E-mail: ampomahbrown@yahoo.com

\section{Summary}

Objective. The purpose of this study was to identify the various patterns of insertion of the anterior horn of the medial meniscus in Ghanaian subjects.

Study. The study involved 35 cadaveric knees (26 males and 9 females). Berlet and Fowler classification was used to classify the insertion of the anterior horn of the medila meniscus.

Findings. The distribution of the insertion pattern was as follows; $42.9 \%$ (15) had type I insertion, $45.7 \%$ (16) had type II, type III and IV insertions were each found in $5.7 \%$ (2) of the dissected knees. Type II insertion had the highest incidence which was a deviation from what has been reported in literature. The incidence of the anterior intermeniscal ligament (AIML) was $34.3 \%$, which was much lower that most studies have reported. Conclusion. The findings of the study may suggest that the pattern of insertion of the anterior horn of the medial meniscus may be different in the Ghanaian population; further research is needed in this area.

KEY WORDS: medial meniscus, anterior intermeniscal ligament.

\section{Introduction}

The menisci are crescentic plates of fibrocartilage located on the articular surface of the tibial plateaus of the knee joint and their presence is vital for proper function of the joint. They give stability to the joint and help distribute loads by increasing the congruence of the articulation between the tibia and the femur $^{1,2}$. They also have propioceptive function and may possibly assist in joint lubrication ${ }^{1}$.
Extrusion of the menisci by the femoral condyles is mainly resisted by its firm anchorage to the tibia bone particularly at the menisci horns, hence the integrity of the insertion of the menisci are crucial for proper function. According to Gray's anatomy ${ }^{1}$ the anterior horn of the medial meniscus in the adult knee is usually attached to the anterior tibial intercondylar area in front of the anterior cruciate ligament.

Though some studies have found this to be the case ${ }^{3-5}$ other sites of insertions have also been identified. This includes insertion onto other areas of the medial tibial plateau aside the flat anterior intercondylar area ${ }^{3}$, as well as insertions onto the anterior cruciate ligament $(A C L)^{3-8}$ the anterior intermeniscal ligament $(A I M L)^{3-6}$ and infrapatellar synovial fold 6 . Some studies have even described insertions onto the intercondylar notch of femur ${ }^{9-11}$. The insertion of the anterior horn of the medial meniscus (MM) have over a period of time been studied by direct observation and by imaging techniques, however there seems to be no information on the various sites of insertion in the Ghanaian population. The purpose of this study was to identify the various patterns of insertions of the anterior horn of the MM in Ghanaian specimens.

\section{Materials and methods}

The study was conducted in the Department of Anatomy of the School of Medical Sciences, University of Cape Coast using 35 cadaveric knees (16 paired and 3 unpaired) from 14 males and 5 females.

The knee joint was opened by first removing the skin around the knee and transecting the quadriceps femoris muscle proximal to the patella and reflecting the patella inferiorly. The medial and lateral collateral ligaments were then released; the anterior and posterior cruciate ligaments transected and the knee gently flexed to expose the menisci. The different insertion sites of the anterior horn of the medial meniscus were noted and classified using the classification system described by Berlet and Fowler. This system classifies the insertion from type I to IV as follows:

Type I-insertion is located in the flat intercondylar region of the tibial plateau.

Type II-insertion is on the downward slope from the medial articular plateau to the intercondylar region.

Type III-insertion is on the anterior slope of the medial tibial plateau.

Type IV-insertion has no firm attachment of the meniscus and there is no identifiable area on the tibial plateau where it attaches. 
The anterior horn was also examined for any structural connection with the ACL (menisco-cruciate association). The anterior fat pad was later dissected to determine the presence of anterior intermeniscal ligament (AIML).

\section{Results}

The total number of knees dissected was 35 , of these $33(94.3 \%)$ had the anterior horns of their medial menisci anchored to an area on the tibial plateau. Two knees $(5.7 \%)$ however had no identifiable attachment of their anterior horns to any part of the tibial plateau. The distribution of insertion patterns from the study was as follows: $42.9 \%$ (15) had type I insertion, $45.7 \%$ (16) had type II, types III and IV insertions were each found in $5.7 \%$ (2) of the dissected knees. With the exception of one specimen all paired knees demonstrated similar pattern of insertion. The AIML was present in 12 (34.3\%) knees and association between the anterior horn and the ACL was observed in $20(57.1 \%)$ knees (Tab. 1), (Figs. 1,2).

\section{Discussion}

In our study $42.9 \%$ (15) of dissected knees had the anterior horns of their MM inserted on to the flat anterior intercondylar area of the tibial plateau (Type I). The most common site of insertion however was on the area of downward slope between the medial articular plateau and the intercondylar area (Type II) and this insertion pattern was observed in $45.7 \%$ (16) of the knees. More that $50 \%$ of the knees had insertion site other than the site generally described in literature. This differs from the findings of Berlet and Fowler ${ }^{3}$ who observed type I insertion pattern in 59\% of specimen. This deviation from the norm may suggest that in some populations the flat anterior intercondylar area may not the most common site of insertion of the anterior horn of the medial meniscus. Types III and IV insertion patterns each had an incidence of $5.7 \%$ and were observed in paired knees. There was one cadaver in which the pattern of insertion was not identical bilaterally. The right knee had a type I while the left had type II insertion pattern.

The function of the AIML is not fully understood it has however been suggested that it may have biomechanical $^{12}$ as well as proprioceptive functions ${ }^{3}$.

In our study $12(34.3 \%)$ of cadavers had the AILM. This number appears small in comparison to the findings of other cadaveric studies which have reported an incidence between 55\% and 94\% $3,5,13-16$. There was a $100 \%$ incidence of the AIML in both type III and type IV insertions a finding that is consistent with the observations of Berlet and Fowler ${ }^{3}$. Suggesting that, the presence of the AIML may be important in maintaining the functional integrity of the menisci in these particular types of insertions.

Studies have also identified a structural link between the ACL and the anterior horn of the medial meniscus $^{3,5}$, and it is speculated that the connection of fibers from the anterior horn to the ACL could serve as a secondary restraint to motion of the anterior horn of the medial meniscus ${ }^{5}$.

Table 1. Incidence of AIML and Menisco-Cruciate association in the various insertion Types.

\begin{tabular}{lll}
\hline Type of insertion & Menisco-cruciate Association (\%) & AIML (\%) \\
\hline I & $10(66.7)$ & $3(20.0)$ \\
II & $8(50.0)$ & $5(31.3)$ \\
III & $2(100.0)$ & $2(100.0)$ \\
IV & $0(0.0)$ & $2(100.0)$ \\
\hline
\end{tabular}

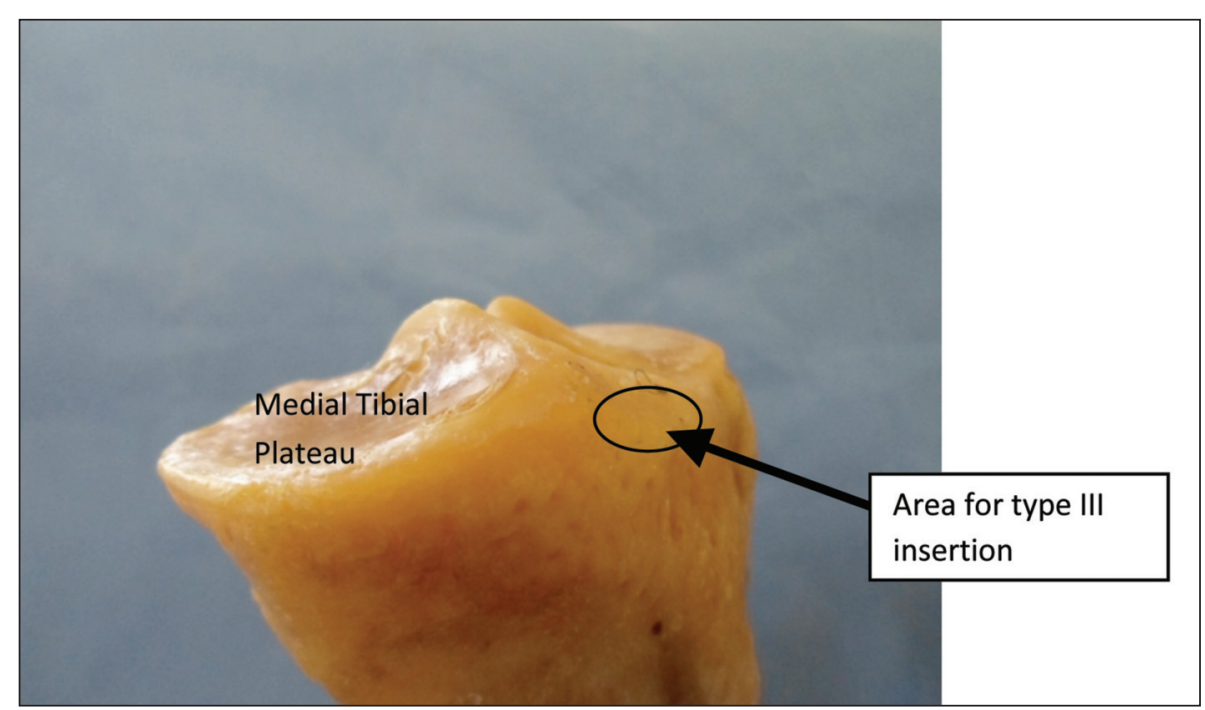

Figure 1. Type III insertion site of the anterior horn of the medial meniscus. 


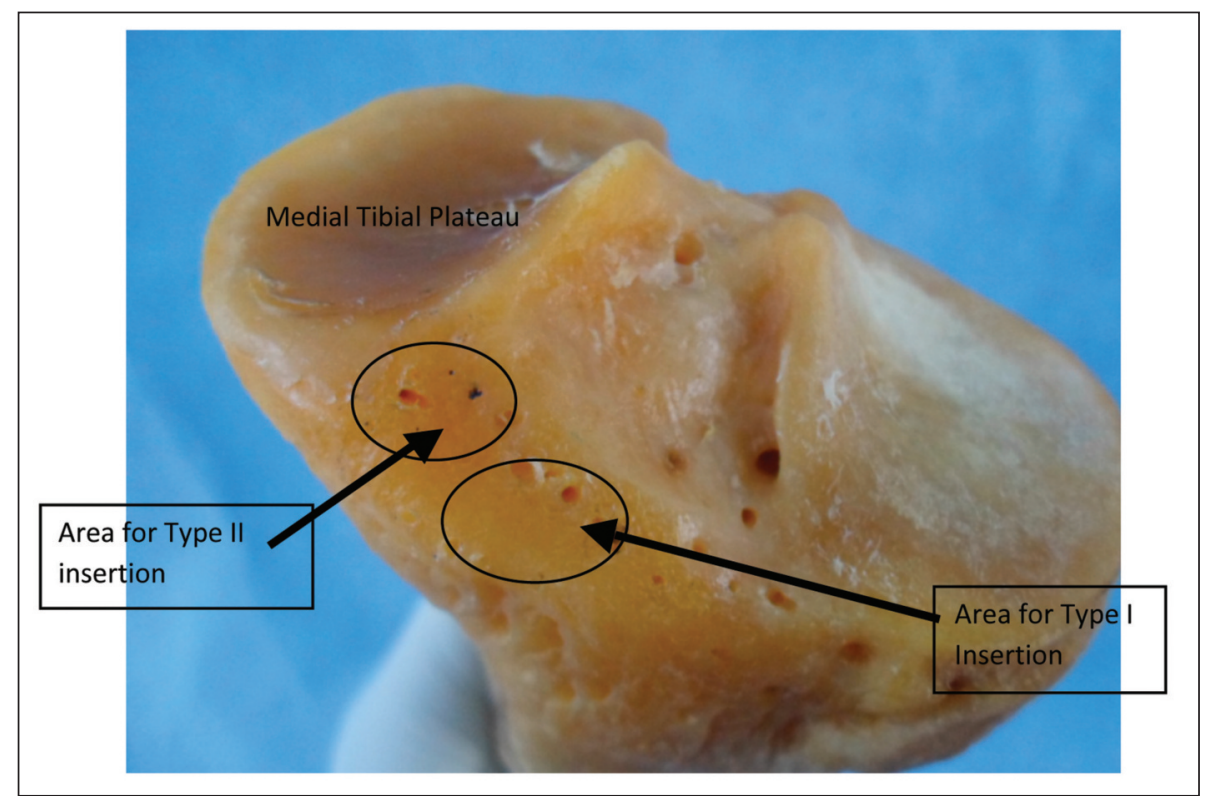

Figure 2. Type I and II insertion sites of the anterior horn of the medial meniscus.

Kohn and Moreno ${ }^{5}$ reported this association in all specimens dissected. This was however not the observation in this study which found its occurrence in only $57.1 \%$ of the dissected knees, a finding that is similar to $59 \%$ incidence reported by Berlet and Fowler ${ }^{2}$.

\section{Conclusion}

The findings of our study are suggestive of two things: 1) that the flat area of the anterior intercondylar area may not be the most common site for insertion of the anterior horn of the medial among the Ghanaian population; 2) that the incidence of the AIML may also be much lower among Ghanaians. Care must however be taking when extrapolating the findings of this study to the general population due of the small sample size involved. Not withstanding, these findings seem to suggest a deviation from the expected and need further investigation.

\section{Reference}

1. Stranding S. Gray's anatomy: Anatomical basis of clinical practice 39th ed. Elsevier Churchill Livingstone 2005; 14761477.

2. de Albornoz PM, Forriol F. The meniscal healing process. Muscles Ligaments Tendons J 2012; 2(1):10-18.

3. Berlet GC, Fowler PJ. The anterior horn of the medial meniscus. An anatomic study of its insertion. Am J Sports Med 1998; 26:540-543.

4. Kale A, Kopuz C, Dikici F, et al. Knee Surg Sports Traumatol Arthrosc 2010; 18:754-759.
5. Kohn D, Moreno B. Meniscus insertion anatomy as a basis for meniscus replacement: a morphological cadaveric study. Arthroscopy 1995; 11:96103.

6. Ohkoshi Y, Takeuchi T, Inoue C, et al. Arthroscopic studies of variants of the anterior horn of the medial meniscus. Arthroscopy 1997; 13:725-730.

7. Rainio P, Sarimo J, Rantanen J, et al. Observation of anomalous insertion of the medial meniscus on the anterior cruciate ligament. Arthroscopy 2002; 18(2):9.

8. Cha JG, Min KD, Han JK, et al. Anomalous insertion of the medial meniscus into the anterior cruciate ligament: the MR appearance. Br J Radiol 2008; 81:20-24.

9. Jung YB, Yum JK, Bae YJ, et al. Anomalous insertion of the medial menisci. Arthroscopy 1998; 14:505-507.

10. Arjun S, Takahashi S, Tang Y, et al. MR appearance of anomalous insertion of the medial meniscus A case report. Acta Radiologica 1998; 39(5):554-556.

11. Shea KSG, Westin C, West J. Anomalous insertion of the medial meniscus of the knee. A case report. J Bone Joint Surg Am 1995; 77:1894-1896.

12. Muhle C, Thompson WO, Sciulli R, et al. Transverse ligament and its effect on meniscal motion: Correlation of kinematic MR imaging and anatomic sections. Invest Radiol 1999 34:558-565.

13. Tubbs RS, Mechelson J, Loukas M, et al. The transverse genicular ligament: anatomical study and review of the literature. Surg Radiol Anat 2008; 30(1):5-9.

14. Zivanovic S. Menisco-meniscal ligaments of the human knee joint. Anat Anz 1974; 135:35-42.

15. Nelson EW, LaPrade RF. The Anterior Intermeniscal Ligament of the Knee An Anatomic Study. Am J Sports Med 2000; 28(1):74-76.

16. Marcheix PS, Marcheix B, Siegler J, et al. The anterior intermeniscal ligament of the knee: an anatomic and MR study. Surgical and radiologic anatomy 2009; 31(5):331334. 